\title{
Ion-induced Auger Electron Spectroscopy as a Potential Route to Chemical Focused-Ion Beam Tomography
}

\author{
Hamed Parvaneh $^{1}$, Robert Hull ${ }^{1}$ \\ 1. Department of Materials Science and Engineering, Rensselaer Polytechnic Institute, Troy, New York \\ 12180, USA
}

In this paper the possible application of ion-induced Auger electron spectroscopy (IAES) for 3D chemical tomography using the focused ion beam (FIB) is discussed. In conventional electron-induced Auger electron spectroscopy (EAES), an electron beam with energy $1-10 \mathrm{kV}$ is used to excite inner-shell (core) electrons of the solid. An electron from a higher electron energy state de-excites to this hole and the extra energy is then transferred to another electron, i.e. the Auger electron, which has a characteristic energy and serves to identify the excited atom [e.g. 1, 2]. In IAES, on the other hand, very large excitation cross sections can occur by promotion of inner shell electrons through crossing of molecular orbitals. Such excitation processes have been described by Barat and Lichten [3] for bi-particle gas phase collisions. In addition to excitation of incident or target atoms, there would also be a substantial momentum transfer from the incident to the target atoms, which may cause the target atom to sputter off leading to the possibility of decay while the atom is either in motion in the solid or is traveling in vacuum after sputtering [e.g. 4]. As a result there are differences between spectra induced by electrons and ions. In particular, interpretation of the ion-induced Auger spectra requires separate consideration of both excitation and decay processes.

We have successfully integrated a state-of-the-art mass-filtered FIB (Orsay Physics) with a PHI Versaprobe XPS instrument. The concentric hemispherical analyzer (CHA) of the Versaprobe is then utilized to measure the kinetic energy of the Auger electrons induced by the ions from a gold-silicon alloy source. IAE spectra of some of the elements in third-row of the periodic table, namely $\mathrm{Na}, \mathrm{Mg}, \mathrm{Al}$, $\mathrm{Si}$, and the ones in the fourth-row, namely $\mathrm{Ti}, \mathrm{V}, \mathrm{Cr}, \mathrm{Mn}, \mathrm{Fe}, \mathrm{Co}, \mathrm{Ni}$ and $\mathrm{Cu}$ acquired using $\mathrm{Si}^{++}$and $\mathrm{Au}^{+}$ incident ions will be presented and discussed (An example for $\mathrm{Cr}$ is shown in Figure 1, left). As a result of energetic collisions between the incident and target atoms, in addition to plasmon excitations, Auger electrons from both colliding particles are generated and detected. The efficiencies of Auger electron generation by ion impact from these elements, acquired under the same conditions, are compared with each other. The elements on the third row of periodic table in particular show narrow peaks emanated mainly from the decay of excited atoms. For heavier elements, however, the increase of fluorescence yield by increasing atomic number results in reduction of atomic contribution to the spectrum. Nevertheless, by using optimized analyzer settings, sharper peaks with higher signal to noise ratio are also achieved for these elements that sets the foundation for chemical tomographic imaging using IAE spectroscopy.

This work was supported through NYSTAR C080117 (research) and NSF-MRI 0923181 (instrumentation) 


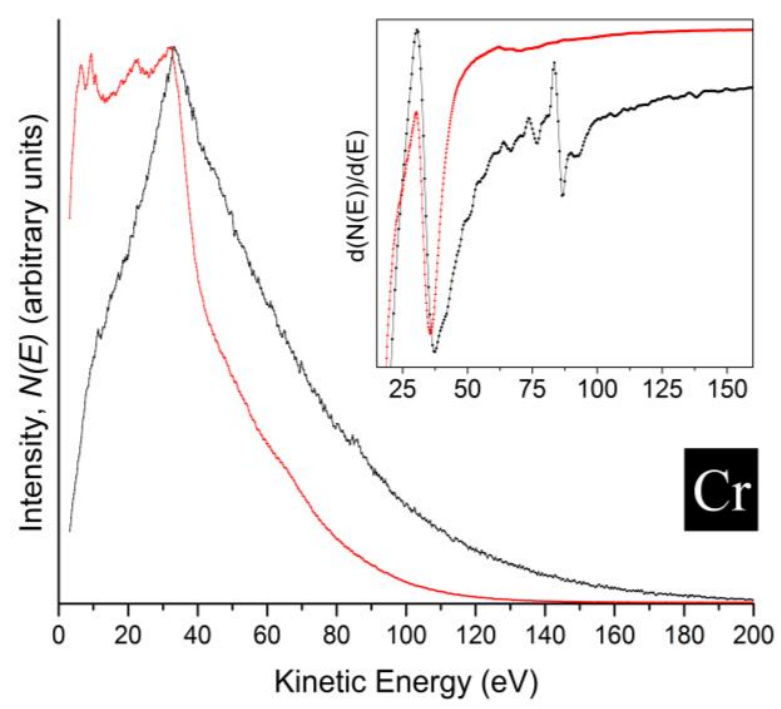

Figure 1. The IAE spectrum of $\mathrm{Cr}$ induced by $\mathrm{Si}^{++}$(black) and $\mathrm{Au}+$ (red) incident ions. The inset shows an spectrum acquired using high pass energy in differentiated mode.

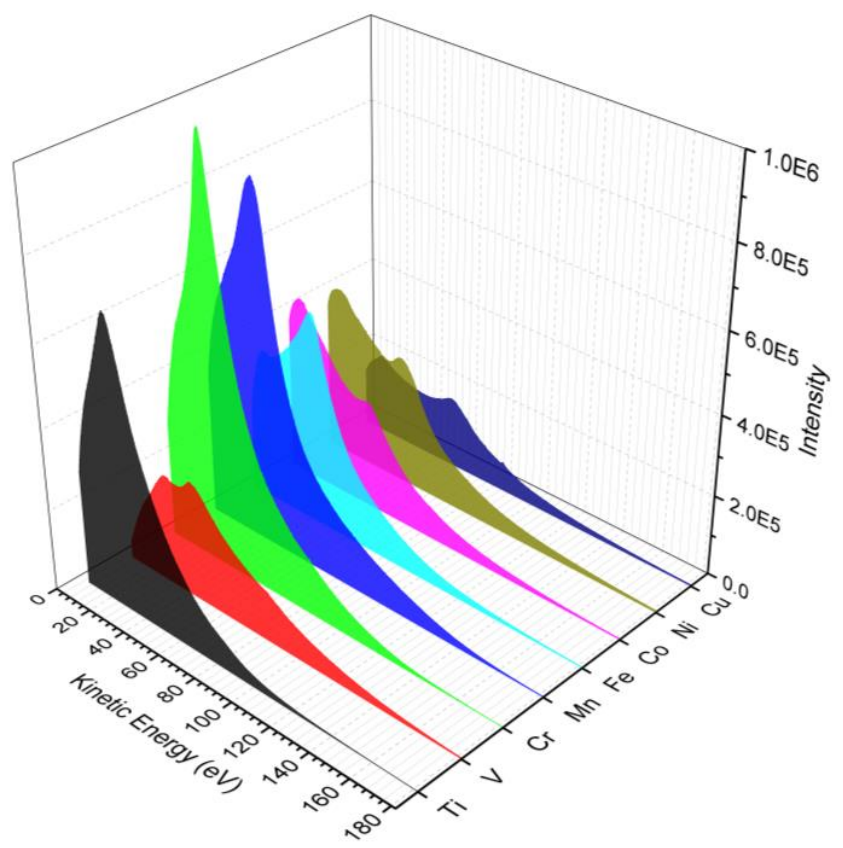

Figure 2. Comparison of relative counts of IAE spectra for several elements using $\mathrm{Si}^{++}$incident ions and similar acquisition conditions.

References:

[1] J. T. Grant in "Characterization of Metal and Polymer Surfaces", ed, L. H. Lee, (Academic, N.Y., Vol. 1, 1977) p. 133.

[2] T. E. Gallon in "Electron and Ion Spectroscopy of Solids", ed. L. Fiermans, J. Vennik, and W.

Dekeyser, (Plenum, N.Y., 1978) p. 230.

[3] M. Barat and W. Lichten, Phys. Rev. A 6, (1972), p. 211.

[4] J. S. Briggs, Rep. Prog. Phys. 39 (1975), p. 217-289.

[5] R. A. Baragiola, Radiation Effects 61 (1982) P. 47-72. 